\title{
Modelagem e Controle de um Robô Auto-Equilibrante Segway
}

\author{
Guilherme Elci Tamiozzo *, Rômulo Lira Milhomem.* \\ *Loboratório de Instrumentação e Controle - LABICON, Instituto Federal de Santa Catarina - IFSC, Chapecó, SC, \\ (e-mail:gui.elci@unochapeco.edu.br,romulo.milhomem@ifsc.edu.br).
}

\begin{abstract}
The paper presents the mathematical modeling of a PISDR, as well as the design and implementation of two linear controller architectures. The dynamic equations of the system were obtained according to the formulation of the Euler-Lagrange equations, the implemented control architectures were based on Lyapunov's asymptotic stability theory and the classical control theory, respectively. The practical implementations of the controllers allowed the obtaining of the dynamic response of the plant in two different test scenarios: in the first, the initial condition response was studied by specifying an initial state vector, in the second, the response of the variables was analyzed. of system states at a prototype position reference step. For both implementations, simulation and experimental results are presented, showing the functioning of the proposed prototype.
\end{abstract}

Resumo: O trabalho apresenta a modelagem matemática de um PISDR, além do projeto e implementação de duas arquiteturas de controladores lineares. As equações dinâmicas do sistema foram obtidas segundo a formulação das equações de Euler-Lagrange, as arquiteturas de controle implementadas foram baseadas na teoria de estabilidade assintótica de Lyapunov e na teoria clássica de controle, respectivamente. As implementações práticas dos controladores permitiram a obtenção da resposta dinâmica da planta em dois cenários de testes diferentes: no primeiro, estudou-se à resposta a condição inicial, especificando-se um vetor de estado inicial, no segundo, analisou-se à resposta das variáveis de estados do sistema a um degrau de referência de posição do protótipo. Para ambas as implementações são apresentados resultados de simulações e experimentais, mostrando o funcionamento do protótipo proposto.

Keywords: Inverted Pendulum on Two Wheels, Euler-Lagrange Equations, Control Theory.

Palavras-chaves: Pêndulo Invertido Sobre Duas Rodas, Equações de Euler-Lagrange, Teoria de Controle.

\section{INTRODUÇÃO}

Sistemas do tipo Pêndulo Invertido são tema de estudo recorrente na área da Engenharia de Controle e Sistemas, os quais são aplicados ao desenvolvimento de robôs caracterizados como Pêndulos Invertidos Sobre Duas Rodas (PISDR). Um PISDR consiste em uma haste com liberdade para girar, ligada a duas rodas atuadas por motores, caracterizando o dispositivo conhecido como Segway (Segway Inc., 2011). O objetivo principal no controle de um PISDR é manter o pêndulo na posição vertical e as rodas do mesmo em repouso (Nawawi et al., 2008).

Por se tratar de um sistema intrinsicamente instável, com dinâmica não linear, PISDR constituem excelentes plataformas de teste e de prova de diversas estratégias de controle, como em Vendramini e Silva (2010) que trabalharam na estabilização de um sistema semelhante utilizando técnicas de controle clássico e adaptativo. Assim, um critério importante no projeto de controle de um PISDR é o de estabilidade, que pode ser validado a partir do método de Lyapunov, útil na determinação da estabilidade de sistemas lineares, não lineares e/ou variantes no tempo (Ogata, 2011), permitindo assim, o projeto e a implementação de controladores lineares. Neste sentido, apresentam-se duas arquiteturas de controle aplicadas a um PISDR desenvolvido no Laboratório de Instrumentação e Controle. A primeira arquitetura é baseada em condições de equilíbrio segundo a teoria de estabilidade de Lyapunov. A segunda é baseada em um arranjo de controladores do tipo Proporcional Integral Derivativo (PID). Para isso, também é apresentado a modelagem dinâmica de um PISDR via equações de EulerLagrange. A seguir, será apresentada a modelagem matemática do sistema com base na formulação das equações de Euler-Lagrange, assim como a descrição dos parâmetros físicos do modelo e a validação matemática das equações dinâmicas da planta.

\section{MODELAGEM DINÂMICA}

O sistema físico proposto para o protótipo de equilíbrio Segway é ilustrado na Figura 1, onde o pêndulo invertido é montado sobre um suporte fixado aos motores que promovem a movimentação do robô. Os componentes 1 e 2 descritos na Figura 1 denotam respectivamente o corpo inferior (conjunto motor elétrico e rodas) e corpo superior (haste invertida). Para efeitos de nomenclaturas, cálculos e determinação da correlação física entre as variáveis que compõem o modelo físico do sistema, considere a descrição do Quadro 1. A dinâmica do robô de equilíbrio Segway é obtida através da formulação das equações de Euler-Lagrange adaptadas com a inclusão da função de dissipação de Rayleigh. Para tanto, emprega-se o cálculo da energia cinética e potencial do sistema físico, além da energia advinda de forças de atrito viscoso proporcionais às velocidades dos corpos.

A energia cinética do corpo inferior é dada por (1). Uma vez especificada a relação de redução do motor $K_{r}$ e considerando 
que a roda rola sem deslizar, é possível definir relações de transformação do movimento rotacional em translacional, por $\theta_{r}=\frac{x}{r}, \theta_{m}=\frac{K_{r} x}{r}$.

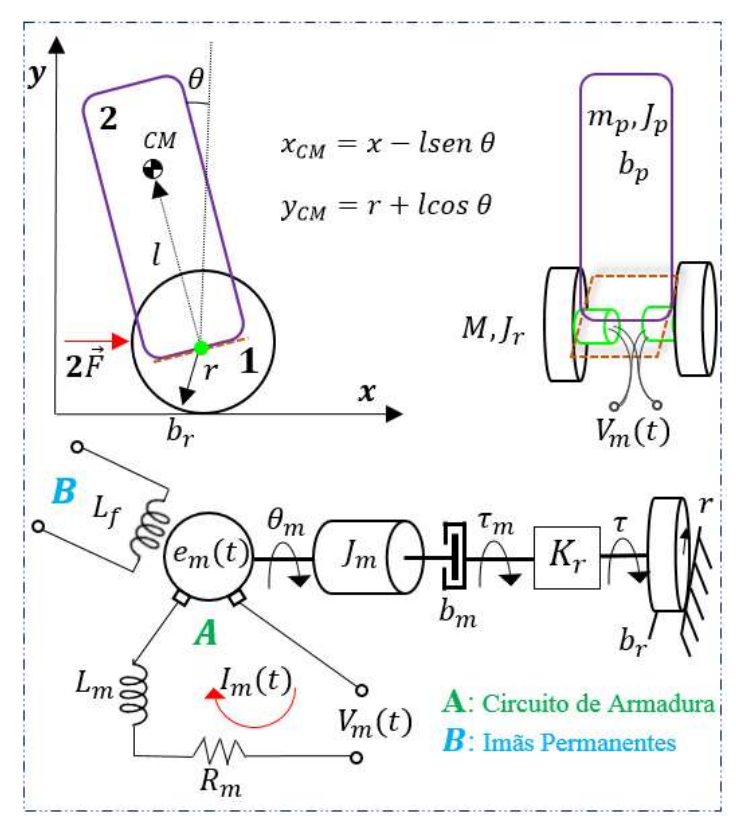

Figura 1. Modelo físico do pêndulo invertido e motores.

Quadro 1. Variáveis e constantes do sistema.

\begin{tabular}{|c|l|}
\hline Símbolo & \multicolumn{1}{c|}{ Descrição } \\
\hline$\theta$ & Ângulo do pêndulo com a vertical \\
\hline$m_{p}$ & Massa global do pêndulo \\
\hline$M$ & Massa global do corpo inferior \\
\hline$J_{p}$ & $\begin{array}{l}\text { Momento de inércia do pêndulo em relação ao centro de } \\
\text { massa }\end{array}$ \\
\hline$J_{r}$ & Momento de inércia da roda \\
\hline$J_{m}$ & Momento de inércia do motor \\
\hline$b_{p}$ & Coeficiente de atrito viscoso do pêndulo \\
\hline$b_{r}$ & Coeficiente de frição da roda \\
\hline$b_{m}$ & Coeficiente de atrito viscoso do motor \\
\hline$g$ & Aceleração da gravidade \\
\hline$l$ & Distância do eixo ao centro de massa do pêndulo \\
\hline$F$ & Força aplicada por um motor \\
\hline$r$ & Raio da roda \\
\hline$V_{m}(t)$ & Tensão elétrica na armadura \\
\hline$I_{m}(t)$ & Corrente elétrica na armadura \\
\hline$R_{m}$ & Resistência elétrica do motor \\
\hline$L_{m}$ & Indutância do motor \\
\hline$e_{m}(t)$ & Força contra-eletromotriz induzida \\
\hline$K_{r}$ & Relação de redução motor/caixa \\
\hline$\theta_{m}$ & Posição angular do motor \\
\hline$\theta_{r}$ & Posição angular da roda \\
\hline
\end{tabular}

$$
k_{1}=\frac{1}{2} M(\dot{x})^{2}+2 \frac{1}{2} J_{m}\left(\dot{\theta}_{m}\right)^{2}+2 \frac{1}{2} J_{r}\left(\dot{\theta}_{r}\right)^{2} .
$$

Desse modo, (1) pode ser reescrita como (2):

$$
k_{1}=\frac{1}{2}\left[M+\frac{2\left(J_{m} K_{r}{ }^{2}+J_{r}\right)}{r^{2}}\right](\dot{x})^{2} .
$$

A energia cinética do pêndulo segue o mesmo procedimento de análise, sendo

$$
k_{2}=\frac{1}{2} m_{p}\left(v_{C M}\right)^{2}+\frac{1}{2} J_{p}(\dot{\theta})^{2},
$$

onde $v_{C M}$ representa a velocidade linear do Centro de Massa $(C M)$ do pêndulo invertido. O módulo de $v_{C M}$ pode ser obtido pela relação $v_{C M}{ }^{2}=\dot{x}_{C M}{ }^{2}+\dot{y}_{C M}{ }^{2}$. Assim a parcela inicial de energia cinética do pêndulo fornecida pelo primeiro termo em (3) provê:

$$
\frac{1}{2} m_{p}\left[(\dot{x})^{2}-2 \dot{x} \dot{\theta} l \cos \theta+(\dot{\theta} l)^{2}\right] \text {. }
$$

A energia potencial do corpo inferior $u_{1}$ é zero, uma vez que por convenção o centro de massa do mesmo assume o nível de referência nula de energia potencial. Por consequência, a energia potencial do pêndulo invertido $u_{2}$ é diretamente proporcional à distância ao nível zero de energia potencial: $u_{1}=0$ e $u_{2}=m_{p} g l \cos \theta$. Ademais a função em energia de Lagrange $L$ pode ser obtida combinando as relações $u_{1} \mathrm{e} u_{2} \mathrm{e}$ (2), (3) e (4):

$$
L=\left(k_{1}+k_{2}\right)-\left(u_{1}+u_{2}\right) \text {. }
$$

Conseguintemente a dinâmica do robô de equilíbrio é determinada através da formulação das equações de movimento de Lagrange que estabelece:

$$
\frac{d}{d t}\left(\frac{\partial L}{\partial \dot{\theta}}\right)-\frac{\partial L}{\partial \theta}+\frac{\partial F_{R a y}}{\partial \dot{\theta}}=\Gamma,
$$

onde $\theta$ corresponde ao vetor de coordenadas independentes do sistema, $\Gamma$ ao vetor de forças aplicadas e $F_{R a y}$ a soma escalar composta por funções de dissipação de Rayleigh. Para o sistema físico proposto a função de dissipação de Rayleigh pode ser definida por:

$$
F_{\text {Ray }}=\frac{1}{2}\left[b_{p}(\dot{\theta})^{2}+2 b_{r}(\dot{x})^{2}+2 b_{m}\left(\dot{\theta}_{m}\right)^{2}\right] .
$$

Substituindo (5) e (7) em (6) obtêm-se:

$$
\begin{gathered}
\left(M_{e q}+m_{p}\right) \ddot{x}-l m_{p}(\ddot{\theta} \cos \theta) \\
+l m_{p}\left(\dot{\theta}^{2} \operatorname{sen} \theta\right)+2\left(b_{r}+\frac{b_{m} K_{r}^{2}}{r^{2}}\right) \dot{x}=2 F . \\
\left(m_{p} l^{2}+J_{p}\right) \ddot{\theta}-l m_{p}(\ddot{x} \cos \theta) \\
-l m_{p} g \operatorname{sen} \theta+b_{p} \dot{\theta}=0
\end{gathered}
$$

onde $M_{e q}$ corresponde a massa equivalente:

$$
M_{e q}=\left[M+\frac{2\left(J_{m} K_{r}{ }^{2}+J_{r}\right)}{r^{2}}\right] .
$$

As equações eletromecânicas típicas de um motor elétrico de corrente contínua fornecem:

$$
e_{m}(t)=K_{g} \dot{\theta}_{m}, \tau_{m}(t)=K_{t} \eta_{m} I_{m}
$$


onde $K_{g}$ é a constante de força contra-eletromotriz, $K_{t}$ é a constante de torque, $\eta_{m}$ a eficiência elétrica e $\tau_{m}$ o torque produzido pelo motor. A partir do modelo elétrico do sistema físico disposto na Figura 1, a aplicação de lei de Kirchoff ao circuito de armadura do motor elétrico sob condições de regime estacionário, estabelece que:

$$
\left.I_{m}(t)\right|_{i \approx 0}=\frac{1}{R_{m}}\left(V_{m}(t)-e_{m}(t)\right)
$$

Após alterado pela caixa transmissão com constante de redução $K_{r}$ e eficiência $\eta_{r}$ o torque $\tau$ responsável por aplicar a força

$$
F=\frac{\eta_{r} K_{r} \tau_{m}}{r}
$$

compreende a ação que promove o movimento do sistema. Utilizando as relações de $\theta_{r}$ e $\theta_{m},(11),(12)$ e (13) obtêm-se a força aplicada em função da entrada em tensão $V_{m}(t)$ e da velocidade $\dot{x}(t)$, da forma:

$$
F=\underbrace{\frac{K_{r} \eta_{r} K_{t} \eta_{m}}{r R_{m}}}_{A_{e}}\left(V_{m}(t)-\frac{K_{g} K_{r}}{r} \dot{x}\right) .
$$

Efetuando a substituição de (14) em (8) obtêm-se as equações diferenciais não lineares que caracterizam o comportamento das variáveis de posição linear $x(t)$ das rodas do robô e angular do pêndulo $\theta(t)$ :

$$
\begin{gathered}
\left(M_{e q}+m_{p}\right) \ddot{x}-\operatorname{lm}_{p}(\ddot{\theta} \cos \theta) \\
+\operatorname{lm}_{p}\left(\dot{\theta}^{2} \operatorname{sen} \theta\right)+B_{e q} \dot{x}=2 \cdot A_{e} V_{m}(t), \\
\left(m_{p} l^{2}+J_{p}\right) \ddot{\theta}-l m_{p}(\ddot{x} \cos \theta) \\
-l m_{p} g \operatorname{sen} \theta+b_{p} \dot{\theta}=0,
\end{gathered}
$$

onde o coeficiente de atrito viscoso equivalente do sistema é determinado pela relação (17). Para linearização de (15) e (16) emprega-se aproximações em séries de Taylor até a primeira ordem em torno de $\theta_{0}=0$ como ponto de operação, tem-se: $\operatorname{sen} \theta=\theta, \quad \cos \theta=1$ e $(\dot{\theta})^{2}=0$.

$$
B_{e q}=2\left(b_{r}+\frac{b_{m} K_{r}^{2}}{r^{2}}+A_{e} \frac{K_{g} K_{r}}{r}\right)
$$

Realizando as devidas substituições em (15) e (16) e reescrevendo as equações de movimento em um sistema $S_{1}$, obtêm-se:

$$
S_{1}:\left\{\begin{array}{c}
P \ddot{x}+B_{e q} \dot{x}-L \ddot{\theta}=2 A_{e} V_{m}(t) \\
J_{e q} \ddot{\theta}+b_{p} \dot{\theta}-L g \theta-L \ddot{x}=0
\end{array},\right.
$$

donde à cunho de simplificações, seja: $L=m_{p} l, J_{e q}=$ $m_{p} l^{2}+J_{p}, P=M_{e q}+m_{p}$.

\subsection{Representação em funções de transferência}

É possível fazer com que cada equação em $S_{1}$ contenha apenas uma derivada de segunda ordem:

$$
\left\{\begin{array}{c}
\ddot{x}=\frac{L^{2} g \theta-J_{e q} B_{e q} \dot{x}-L b_{p} \dot{\theta}+2 A_{e} J_{e q} V_{m}}{q} \\
\ddot{\theta}=\frac{P L g \theta-L B_{e q} \dot{x}-P b_{p} \dot{\theta}+2 L A_{e} V_{m}}{q}
\end{array}\right.
$$

com $q=P J_{e q}-L^{2}$. A transformada de Laplace em (19) permite isolar a equação da dinâmica de cada corpo, relacionado a saída do sistema $(X(s), \Theta(s))$ como uma relação da entrada $V_{m}(s)$ e das funções $\left(\Upsilon_{1}(s), \Upsilon_{2}(s)\right)$ dependentes do vetor de condições iniciais dos estados do sistema $\overrightarrow{\boldsymbol{x}}_{\mathbf{0}}=$ $\left[\begin{array}{llll}x_{0} & \theta_{0} & \dot{x}_{0} & \dot{\theta}_{0}\end{array}\right]$, com $x_{0}=x(0), \theta_{0}=\theta(0), \dot{x}_{0}=\dot{x}(0) \quad \mathrm{e}$ $\dot{\theta}_{0}=\dot{\theta}(0)$.

$$
\left\{\begin{array}{l}
X(s)=G_{1}(s) \mathrm{V}_{\mathrm{m}}(s)+\Upsilon_{1}(s) \\
\Theta(s)=G_{2}(s) \mathrm{V}_{\mathrm{m}}(s)+\Upsilon_{2}(s)^{\prime}
\end{array}\right.
$$

onde, $G_{1}(s) \mathrm{V}_{\mathrm{m}}(s)$ e $G_{2}(s) \mathrm{V}_{\mathrm{m}}(s)$, correspondem a resposta do sistema à condições iniciais nulas, $\overrightarrow{\boldsymbol{x}}_{\mathbf{0}}=\overrightarrow{\mathbf{0}}$.

$$
\left\{\begin{array}{l}
G_{1}=\frac{\left(2 A_{e} / q\right)\left(U_{e q} s^{2}+b_{p} s-L g\right)}{s^{4}+\frac{1}{q}\left(G_{e q} s^{3}+H_{e q} s^{2}-g L B_{e q} s\right)} \\
G_{2}=\frac{\left(2 A_{e} L / q\right) s^{2}}{s^{4}+\frac{1}{q}\left(G_{e q} s^{3}+H_{e q} s^{2}-g L B_{e q} s\right)}
\end{array}\right.
$$

com $G_{e q}=\left(J_{e q} B_{e q}+P b_{p}\right)$ e $H_{e q}=\left(B_{e q} b_{p}-P L g\right)$. E ainda, $\Upsilon_{1}(s)$ e $\Upsilon_{2}(s)$ são funções da forma:

$$
\left\{\begin{array}{l}
\Upsilon_{1}=\frac{x_{0} s^{3}+\left(a_{2} s^{2}+a_{1} s+a_{0}\right) / q}{s^{4}+\frac{1}{q}\left(G_{e q} s^{3}+H_{e q} s^{2}-g L B_{e q} s\right)} \\
\Upsilon_{2}=\frac{\theta_{0} s^{3}+\left(b_{2} s^{2}+b_{1} s+b_{0}\right) / q}{s^{4}+\frac{1}{q}\left(G_{e q} s^{3}+H_{e q} s^{2}-g L B_{e q} s\right)}
\end{array},\right.
$$

com os coeficientes dos numeradores:

$$
\left\{\begin{array}{l}
a_{2}=\dot{x}_{0}+x_{0} G_{e q} ; \quad b_{2}=\theta_{0} G_{e q}+\dot{\theta}_{0} ; \\
a_{1}=P b_{p} \dot{x}_{0}+x_{0} H_{e q}+L^{2} g \theta_{0}-L b_{p} \dot{\theta}_{0} ; \\
b_{1}=B_{e q}\left(\theta_{0} b_{p}+J_{e q} \dot{\theta}_{0}-L \dot{x}_{0}\right) ; \quad b_{0}=0 ; \\
a_{0}=L^{2} g \dot{\theta}_{0}-P L g \dot{x}_{0}-B_{e q} L g x_{0} .
\end{array}\right.
$$

\subsection{Representação em espaço de estados}

O sistema de equações em (18) transcreve a dinâmica do sistema físico, pode-se determinar a representação em espaço de estados equivalente. Para tanto, seja o sistema abaixo:

$$
S=\left\{\begin{array}{l}
\dot{x}=A x+B \boldsymbol{u} \\
y=C x+D \boldsymbol{u}^{\prime}
\end{array}\right.
$$

onde $\boldsymbol{x}$ é o vetor de estado e $\boldsymbol{u}$ é a entrada de controle. No caso do robô Segway, tem-se $\boldsymbol{x}=\left[\begin{array}{cc}x & \dot{x} \dot{\theta}\end{array}\right]^{\prime}$ onde $\boldsymbol{u}=V_{m}$. Os termos de segunda ordem das variáveis dos estados do sistema apresentam sua forma explícita em (19), de modo que: 


$$
\begin{aligned}
A & =\frac{1}{q}\left(\begin{array}{cccc}
0 & 0 & q & 0 \\
0 & 0 & 0 & q \\
0 & L^{2} g & -J_{e q} B_{e q} & -L b_{p} \\
0 & P L g & -L B_{e q} & -P b_{p}
\end{array}\right), \\
B & =\frac{1}{q}\left(\begin{array}{c}
0 \\
0 \\
2 A_{e} J_{e q} \\
2 L A_{e}
\end{array}\right), C^{\prime}=\left(\begin{array}{ll}
1 & 0 \\
0 & 1 \\
0 & 0 \\
0 & 0
\end{array}\right), D=\left(\begin{array}{l}
0 \\
0
\end{array}\right) .
\end{aligned}
$$

As matrizes $C$ e $D$ representam a saída do sistema dinâmico, mensurada por encoders para posição linear do PISDR e por um algoritmo de fusão sensorial para posição angular do pêndulo. Os sensores utilizados na fusão sensorial são giroscópios e acelerômetros e o algoritmo implementado consiste em uma estimação dinâmica de ângulo, a partir da aplicação do filtro de Kalman com base no trabalho de Frizon et al. (2015).

\subsection{Parâmetros do modelo}

Parâmetros como momento de inércia, podem ser obtidos com base em equações presentes na literatura. $\mathrm{O}$ momento de inércia da roda do robô e o momento de inércia do pêndulo em relação ao centro de massa são obtidos pelas aproximações de:

$$
J_{r}=\left(m_{r} r^{2}\right) / 2, J_{p}=\left(m_{h} L_{h}^{2}\right) / 12,
$$

onde $m_{r}$ corresponde a massa da roda, $m_{h}$ a massa da haste invertida e $L_{h}$ ao comprimento total da haste. Entende-se que a haste do robô Segway compreende apenas ao pêndulo invertido, isto é, subtraindo os motores, acoplamentos e rodas. A Tabela 1 apresenta as magnitudes mensuradas e obtidas para os parâmetros do modelo. Os parâmetros $R_{m}, K_{r}, K_{t}$ e $\eta_{m}$ são especificações dadas pelo fabricante do motor elétrico. A eficiência da redução $\eta_{r}$ foi considerado com magnitude de $90 \%$, após consulta a catálogos de outros fabricantes.

A validação do sistema pode ser realizada mediante comparações entre a resposta medida do sistema físico e a simulada, empregando o modelo matemático. Para viabilizar esse procedimento, o robô Segway foi deslocado para um novo ponto de operação, a saber $(x, \theta)=(0, \theta+\pi)$, que difere do anterior, uma vez que representa um ponto de equilíbrio estável. Nessa situação, o PISDR está localizado na posição de equilíbrio vertical para baixo. Naturalmente a versão linearizada das equações em torno do novo ponto de equilíbrio são idênticas as anteriores, exceto por algumas modificações de sinal dos coeficientes de (15) e (16) resultando nas modificações dos sinais termos $L b_{p}, L B_{e q}$ e $P L g$ da matriz A, e do sinal do termo $2 L A_{e}$ da matriz B de (25).

Tabela 1. Constantes do sistema físico.

\begin{tabular}{|c|c|l|}
\hline Constante & Magnitude & Unidade \\
\hline$m_{h}$ & 0,359 & {$[\mathrm{~kg}]$} \\
\hline$m_{r}$ & 0,060 & {$[\mathrm{~kg}]$} \\
\hline$r$ & 0,030 & {$[\mathrm{~m}]$} \\
\hline$m_{p}$ & 0.643 & {$[\mathrm{~kg}]$} \\
\hline$M$ & 0.484 & {$[\mathrm{~kg}]$} \\
\hline
\end{tabular}

\begin{tabular}{|c|c|l|}
\hline$J_{p}$ & 0,0014 & {$\left[\mathrm{~kg} \cdot \mathrm{m}^{2}\right]$} \\
\hline$J_{r}$ & $2,7 \times 10^{-5}$ & {$\left[\mathrm{~kg} \cdot \mathrm{m}^{2}\right]$} \\
\hline$g$ & 9,81 & {$\left[\mathrm{~m} / \mathrm{s}^{2}\right]$} \\
\hline$l$ & 0,07 & {$[\mathrm{~m}]$} \\
\hline$R_{m}$ & 5,88 & {$[\Omega]$} \\
\hline$K_{r}$ & 30 & {$[-]$} \\
\hline$K_{t}$ & 0,0013 & {$[\mathrm{~N} . \mathrm{m} / \mathrm{A}]$} \\
\hline$K_{g}$ & 0,0120 & {$[\mathrm{~V} \cdot \mathrm{s} / \mathrm{rad}]$} \\
\hline$\eta_{m}$ & 0,95 & {$[-]$} \\
\hline$\eta_{r}$ & 0,90 & {$[-]$} \\
\hline
\end{tabular}

Algumas incertezas associadas aos parâmetros do modelo físico alteram significativamente a natureza da resposta do sistema, como coeficientes de atrito viscoso, por exemplo. A etapa de estimação dos parâmetros ainda não conhecidos do modelo, $b_{p}, b_{r}, b_{m}$ e $J_{m}$, foi desenvolvida mediante a realização de simulações computacionais. O sistema físico e o seu modelo foram submetidos a uma mesma tensão de entrada $V_{m}(t)$, com forma de onda quadrada na frequência de $6,54 \mathrm{rad} / \mathrm{s}$ e com amplitude de 2,00 V. Durante a realização das simulações computacionais, as magnitudes de $b_{p}, b_{r}, b_{m}$ e $J_{m}$ foram sendo modificadas de modo a aproximar a resposta do sistema simulado a resposta do sistema real. A Figura 2 ilustra os sinais obtidos utilizando o modelo linear em vermelho e os sinais mensurados do sistema físico em azul ou verde. Assim, as magnitudes correspondentes de $b_{p}, b_{r}, b_{m} \mathrm{e}$ $J_{m}$ são dispostas na Tabela 2.
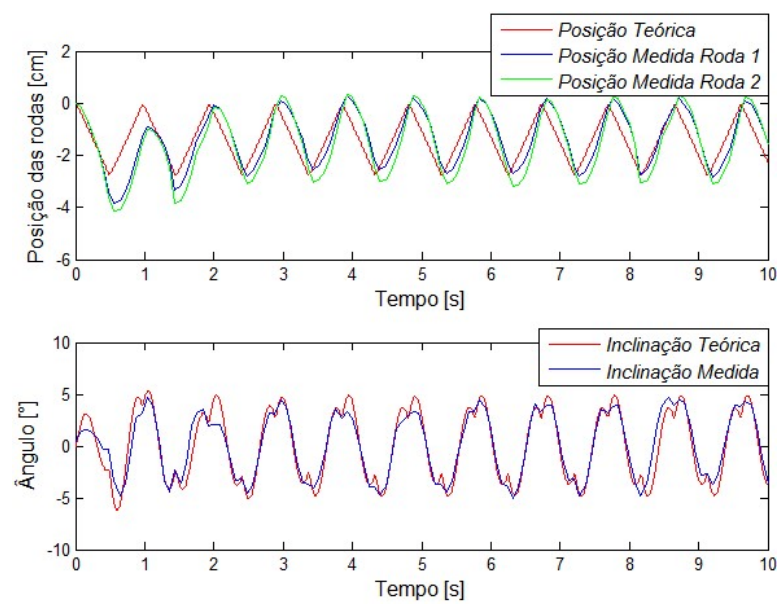

Figura 2. Resposta do sistema para entrada de onda quadrada.

Tabela 2. Descrição de parâmetros.

\begin{tabular}{|c|c|l|}
\hline Constante & Magnitude & \multicolumn{1}{|c|}{ Unidade } \\
\hline$b_{n}$ & 0,00179 & {$[\mathrm{~N} . \mathrm{m} . \mathrm{s} / \mathrm{rad}]$} \\
\hline$b_{r}$ & 0,16 & {$\left[\mathrm{Ns} / \mathrm{m}^{2}\right\rceil$} \\
\hline$b_{m}$ & $2,00 \times 10^{-6}$ & {$[\mathrm{~N} . \mathrm{m} . \mathrm{s} / \mathrm{rad}]$} \\
\hline$I_{m}$ & $0,45 \times 10^{-7}$ & {$\left[\mathrm{~kg} \cdot \mathrm{m}^{2}\right\rceil$} \\
\hline
\end{tabular}

Tabela 3. Métricas comparativas.

\begin{tabular}{|c|c|c|c|c|}
\hline \multirow{2}{*}{ Parâmetro } & \multicolumn{2}{|c|}{ Simulado } & \multicolumn{2}{c|}{ Medido } \\
\cline { 2 - 5 } & $\chi$ & $\theta$ & $\chi$ & $\theta$ \\
\hline$\sigma^{2}$ & 0,81 & 9,69 & 0,96 & 9,40 \\
\hline$\sigma$ & 0,90 & 3,11 & 0,97 & 3,06 \\
\hline RMSE & $0,69 \mathrm{~cm}$ & $1,08^{\circ}$ & - & - \\
\hline
\end{tabular}


Nota-se que o modelo "capturou" adequadamente as características do sistema físico, com leves diferenças em fase, quanto ao deslocamento do robô. Algumas métricas são utilizadas para mensurar a aproximação do modelo teórico ao modelo real, como variância $\sigma^{2}$, desvio padrão $\sigma$ e erro médio quadrático (RMSE-Root Mean Square Error). As magnitudes apresentadas na Tabela 3 representam o quão próximo o modelo teórico corresponde ao modelo real. Nesse caso, a posição do robô mensurada é tomada como a posição linear média das rodas. Assim, observa-se, com base na Tabela 3, que o modelo representa satisfatoriamente o sistema físico estudado. $\mathrm{O}$ erro de desvio padrão de posição foi 7,22 \% a menos do valor mensurado, enquanto que, o erro de desvio padrão do ângulo de inclinação foi de $1,61 \%$ a mais do valor mensurado. O erro médio quadrático obtido entre os modelos revela a natureza similar dos mesmos. A Tabela 4 apresenta as magnitudes equivalentes do sistema físico, constituindo os parâmetros do modelo teórico em (18) e (25).

Tabela 4. Parâmetros equivalentes do sistema.

\begin{tabular}{|c|c|l|}
\hline Constante & Magnitude & Unidade \\
\hline$P$ & 1,2770 & {$[\mathrm{~kg}]$} \\
\hline$B_{e q}$ & 8,9731 & {$\left[\mathrm{Ns} / \mathrm{m}^{2}\right]$} \\
\hline$L$ & 0,0450 & {$[\mathrm{~kg} \mathrm{~m}]$} \\
\hline$A_{e}$ & 0,1939 & {$[\mathrm{~N} / \mathrm{V}]$} \\
\hline$J_{e q}$ & 0,0046 & {$\left[\mathrm{~kg} \cdot \mathrm{m}^{2}\right]$} \\
\hline$q$ & 0,0038 & {$\left[\mathrm{~kg}^{2} \cdot \mathrm{m}^{2}\right]$} \\
\hline$b_{P}$ & 0,00179 & {$[\mathrm{~N} \cdot \mathrm{m} \cdot \mathrm{s} / \mathrm{rad}]$} \\
\hline
\end{tabular}

\subsection{Protótipo do PISDR proposto}

O protótipo desenvolvido apresenta como característica principal seu corpo rígido e um eixo com duas rodas independentes, conforme ilustrado na Figura 3. O protótipo é instrumentado com um drive de potência composto por uma ponte H L298N com tensão de operação na faixa de 6,0 a 35,0 $\mathrm{V}$ e corrente de trabalho máxima de $2,0 \mathrm{~A}$ por canal. Cada qual é conectado a um motor que opera com tensão nominal de 12,0 $\mathrm{V}$ e fornece 320 RPM (rotações por minuto) sem carga imposta ao eixo. Cada motor apresenta uma caixa de redução com fator de 1:30 e um encoder acoplado diretamente ao eixo do mesmo com resolução de 330 Pulso Por Revolução (PPR). A realimentação do ângulo de inclinação com a vertical é um dos estados necessários para o controle do protótipo. Para tal tarefa, o PISDR conta com uma Unidade de Medição Inercial (IMU) disposta no módulo MPU6050. Dois módulos de radiofrequência NRF24L01 são utilizados proporcionando a existência de um canal para a troca de dados entre o PISDR e o sistema de aquisição de dados. No Quadro 2 apresentam-se os componentes.

Quadro 2. Componentes presentes no PISDR.

\begin{tabular}{|c|l|}
\hline$N^{\circ}$ & \multicolumn{1}{|c|}{ Descricão } \\
\hline 1 & Botão de energização do PISDR de dois estados \\
\hline 2 & Conector de alimentação fêmea tipo P4 \\
\hline 3 & Módulo interno de radiofrequência NRF24L01 \\
\hline 4 & Módulo externo de radiofrequência NRF24L01 \\
\hline 5 & Módulo GY-521 - MPU6050 \\
\hline 6 & Plataforma arduino MEGA 2560 \\
\hline
\end{tabular}

\begin{tabular}{|c|l|}
\hline 7 & Plataforma arduino MEGA 2560 \\
\hline 8 & Ponte H L298N \\
\hline 9 & Encoders de 330 PPR \\
\hline 10 & Motores DC12V 320 RPM \\
\hline 11 & Rodas com diâmetro de 6 cm \\
\hline
\end{tabular}

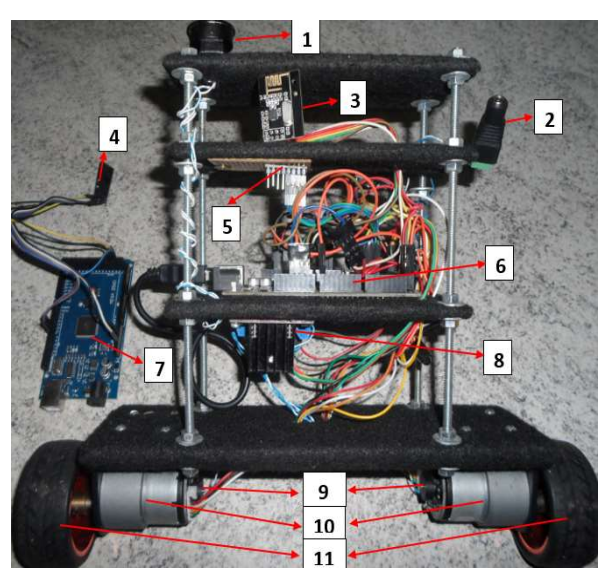

Figura 3. Protótipo experimental do PISDR.

\section{DESENVOLVIMENTO DO SISTEMA DE CONTROLE}

As equações dinâmicas do sistema mostram a correlação existente entre $\theta, x$ e derivadas associadas. Em termos de variáveis controladas de interesse, estão a posição linear média das rodas do protótipo e a posição de inclinação do pêndulo invertido com a vertical. Com base em Vendramini e Silva (2010) foi adotado uma máxima deflexão angular do estado $\theta \approx 3^{\circ}$, assim como um elevado coeficiente de amortecimento para evitar oscilações excessivas, em torno de $0,8<\xi<1$. Além disso, note que os autovalores da matriz $A$ são $\lambda(A)=$ [0 10,7729 - 16,1884 - 5,9062], logo, o sistema é instável em malha aberta, tornando-se necessário utilizar uma lei de controle, de tal modo que o sistema se torne estável.

Para isso foram propostas duas arquiteturas de controle. A primeira segue a realização em espaço de estado para controle da posição angular de inclinação e da posição linear média das rodas do protótipo. A segunda, constitui o projeto de dois controladores do tipo PID (Proporcional Integral Derivativo), um para controle da inclinação do PISDR e outro para posição média das rodas. $\mathrm{Na}$ sequência apresentam-se as suas arquiteturas.

\subsection{Projeto de controle via condições de Lyapunov}

Uma condição necessária e suficiente para estabilidade assintótica de um sistema da forma $\dot{\boldsymbol{x}}=\bar{A} \boldsymbol{x}(t)$, é que exista uma função quadrática dos estados do sistema $V(x(t))=$ $\boldsymbol{x}^{\prime}(t) M \boldsymbol{x}(t)$, com $M=M^{\prime} \in \mathfrak{R}^{n \times n}$ correspondendo a uma matriz definida-positiva, tal que, $\dot{V}(\boldsymbol{x}(t))<0 \forall \boldsymbol{x} \neq 0$ (BOYD et. al., 1994). Assim, tomando a derivada em relação ao tempo de $V(x(t))=x^{\prime}(t) M x(t)$, obtém-se $\bar{A}^{\prime} M+M \bar{A} \prec$ 0 , sendo $M>0$. A matriz $M$ e a função $V(x(t))$ corresponde a matriz de Lyapunov e a função quadrática de Lyapunov, 
respectivamente (Khalil, 2002). Assim, para solução do problema considera-se:

$$
\begin{gathered}
\exists M=M^{\prime}>0, N=N^{\prime}>0: \\
\bar{A}^{\prime} M+M \bar{A}=-N .
\end{gathered}
$$

Assim, se $N$ for definida positiva, então o sistema é assintoticamente estável, sendo as derivadas das trajetórias dos estados nulas em tempo finito, isto é, todos os autovalores de $\bar{A}$ possuirão parte real negativa (Milhomem, 2013). Neste sentido, o projeto de controle tem por objetivo garantir a convergência dos estados do sistema (PISDR) para o seu ponto de equilíbrio, dada uma condição inicial. Para tanto, usa-se o procedimento apresentado em Chen (1999), considerando $F=$ $-\Psi$, a matriz dos autovalores desejados:

$$
\Psi=\left(\begin{array}{cccc}
\xi \omega_{n}+i \omega_{d} & 0 & 0 & 0 \\
0 & \xi \omega_{n}-i \omega_{d} & 0 & 0 \\
0 & 0 & 5 \xi \omega_{n} & 0 \\
0 & 0 & 0 & 5,5 \xi \omega_{n}
\end{array}\right)
$$

com $\xi=0,95$ e frequência natural não amortecida $\omega_{n}=$ 40/19. Ainda, $\omega_{d}=\omega_{n} \sqrt{1-\xi^{2}}$. A resolução da equação de Lyapunov fornece o ganho $K=[-10,9577,64-$ $35,166,60]$. Nesse caso, $\exists M=M^{\prime}>0, N=-F$, $\operatorname{com} \Psi=$ $N=N^{\prime}>0$ tal que:

$$
(A-B K)^{\prime} M+M(A-B K)=-N .
$$

implicando na convergência dos estados para o ponto de equilíbrio do sistema. Os autovalores de $\bar{A}=(A-B K)$ são os elementos dispostos na diagonal de $F$. O controle por realimentação de estado permite fixar a dinâmica da planta através do posicionamento dos pólos desejados (Ogata, 2011).

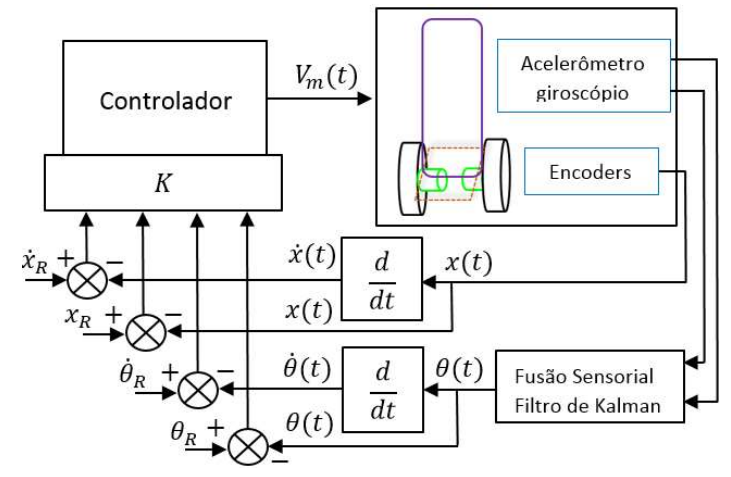

Figura 4. Malha de controle em espaço de estados.

A realimentação de estado, na prática, é obtida com auxílio de dispositivos de instrumentação fixados na planta. A Figura 4 ilustra um diagrama de blocos simplificado, que esquematicamente apresenta o processo de realimentação de estados, a arquitetura de controle e os principais dispositivos de medição utilizados. Os estados mensurados pelos sensores no PISDR correspondem a inclinação angular do pêndulo e posição linear média das rodas. A derivada temporal associada a esses estados fornece as velocidades correspondentes. $\mathrm{Na}$ prática, faz-se uso de um diferenciador digital da forma de (30) (Lathi 2007). Esse diferenciador digital têm a função de estimador de estado, associando a derivada dos estados mensurados.

$$
v_{y}[n T]=\left(\frac{1}{T}\right)(y[n T]-y[(n-1) T]) .
$$

\subsection{Projeto de controle via arquitetura PID}

O sistema de controle proposto é composto por duas malhas, uma contendo o controlador $C_{1}$, responsável pelo controle da posição angular do pêndulo e outra, dispondo do controlador $C_{2}$ para controle da posição linear média das rodas do protótipo, conforme ilustrado na Figura 5.

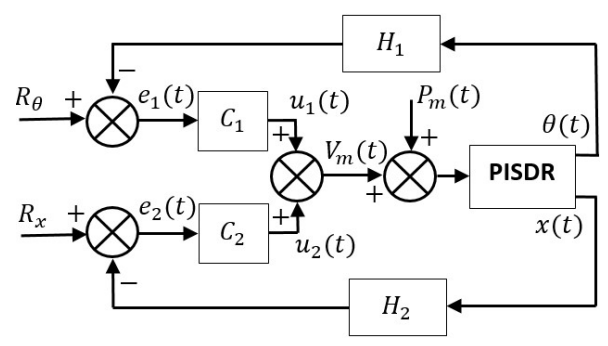

Figura 5. Arquitetura de controle proposta.

Cada uma das malhas possui um sinal de referência $\left(R_{\theta}\right.$ e $\left.R_{x}\right)$ e um sinal de realimentação, que pode ser a própria saída mensurada da planta, ou uma função dela $\left(H_{1}\right.$ e $\left.H_{2}\right)$. No caso em questão, a realimentação é unitária e a referência de inclinação $R_{\theta}$ é nula. Percebe-se que o sinal de atuação $V_{m}(t)$ é regulado pela atuação de dois controladores atuando de forma conjunta. O termo $P_{m}(t)$ corresponde a uma entrada de perturbação em tensão adicionada ao sinal de atuação $V_{m}(t)$. Essa arquitetura é baseada em Jung and King (2008), os quais empregam uma malha de controle para cada saída desejada. Por se tratar de um problema de controle normalmente aplicado a sistemas com múltiplas entradas e múltiplas saídas, a solução proposta consiste em resolver dois subsistemas específicos de controle, cada qual correspondente a saída desejada (Vendramini and Silva, 2010).

$\mathrm{O}$ controlador $C_{2}$ está diretamente relacionado à $C_{1}$, uma vez que essa metodologia de solução pressupõem o encadeamento das ações dos controladores. Inicialmente é necessário equilibrar o PISDR e em seguida, deslocá-lo a partir de uma trajetória de referência (Vendramini and Silva, 2010). Assim, com base em resultados de simulações numéricas, a partir das especificações de desempenho estabelecidas, chegou-se aos seguintes controladores:

$$
C_{i}(s)=\frac{U_{i}(s)}{E_{i}(s)}=P_{i}+\frac{I_{i}}{s}+\frac{D_{i} N_{i} s}{s+N_{i}}, i=1,2 .
$$

onde $P_{1}=110, I_{1}=270, D_{1}=3, N_{1}=N_{2}=100$. E ainda, $P_{2}=100, I_{2}=60$ e $D_{2}=5$. A Equação (31) foi discretizada para a implementação, com período de amostragem $T=0,05$ s. A equação de diferenças em tempo discreto $n$, correspondente a cada controlador $C_{i}(s), i=1,2$ é da forma:

$$
\begin{gathered}
u_{i}[n]=-a_{i} u_{i}[n-1]-b_{i} u_{i}[n-2] \\
+c_{i} e_{i}[n]+d_{i} e_{i}[n-1]+w_{i} e_{i}[n-2] .
\end{gathered}
$$




$$
\left\{\begin{array}{l}
a_{i}=-8 / \Delta_{\mathrm{i}} ; b_{i}=\left(4-2 N_{i} T\right) / \Delta_{\mathrm{i}} \\
c_{i}=\left[4 \Omega_{i}+2 T \Lambda_{\mathrm{i}}+T^{2} N_{i} I_{i}\right] / \Delta_{\mathrm{i}} \\
d_{i}=\left[2 N_{i} I_{i} T^{2}-8 \Omega_{i}\right] / \Delta_{\mathrm{i}} ; \\
w_{i}=\left[4 \Omega_{i}-2 T \Lambda_{\mathrm{i}}+T^{2} N_{i} I_{i}\right] / \Delta_{\mathrm{i}} .
\end{array}\right.
$$

com $\Delta_{\mathrm{i}}=4+2 N_{i} T, \Omega_{i}=P_{i}+D_{i} N_{i}$ e $\Lambda_{\mathrm{i}}=P_{i} N_{i}+I_{i}$.

\section{RESULTADOS}

Os testes realizados com os algoritmos de controle tratam de dois cenários distintos. No primeiro, elucida-se a resposta temporal obtida com a imposição de condições iniciais ao sistema, no segundo, estuda-se o comportamento do sistema especificando-se uma referência constante de posição linear. Para ambos os cenários são apresentados resultados de simulações e experimentais, indicadas nas Figuras de 6 a 9 por: variáveis teóricas, sendo a simulação do modelo (25); variáveis mensuradas, advindas do protótipo da Figura 3.

\subsection{Resposta a condições iniciais}

A convergência assintótica dos estados do sistema para o ponto de equilíbrio pode ser averiguada pela imposição e aplicação de energia potencial ao sistema. No caso do modelo teórico simulado, a aplicação dessa energia é especificada pelas condições iniciais consideradas no problema. Para ambas as arquiteturas de controle apresentadas foram utilizadas as mesmas magnitudes para as variáveis de estado inicial do sistema, a saber, $\boldsymbol{x}_{\mathbf{0}}=\left[\begin{array}{llll}0,1 & \pi / 30 & 0 & 0\end{array}\right]^{\prime}$. Esse estado pode ser conferido ao sistema real, a partir de um deslocamento na posição média das rodas do PISDR em $10 \mathrm{~cm}$ $\mathrm{e}$, inclinação do pêndulo com a vertical em um ângulo de $6^{\circ}$.

A posição linear média inicial do PISDR corresponde a uma contagem incremental equivalente associada aos encoders presente em cada motor. De modo, que essa etapa é resolvida em uma simples atribuição de variável. Já a posição angular de inclinação do pêndulo é obtida diretamente monitorando-se as leituras fornecidas pelo sistema de comunicação disposto no PISDR, de modo que, é suficiente inclinar devidamente o pêndulo para a posição angular requerida. As Figuras 6 e 7 ilustram, respectivamente, a resposta do sistema relacionada com a implementação de ambos os controladores propostos. Em ambos os casos é possível comparar graficamente o comportamento do modelo teórico em relação ao modelo real.

Percebe-se que os controladores responderam devidamente a referência especificada, atingindo a condição de regime permanente, dentro do tempo de convergência de $5 \mathrm{~s}$. Quanto ao comportamento dos estados, é notório inferir maior índice de amortecimento relacionado a implementação do ganho de realimentação de Lyapunov e, consequentemente, menor nível de energia cinética associada a variação dos estados do sistema. Uma elipse centrada na origem e com eixos paralelos aos eixos coordenados, permite a inferência de dois parâmetros de medida, cada qual condizente com a região de convergência dos estados do sistema. As magnitudes de cada semieixo da elipse nas Figuras 6 e 7, definem uma região que apresenta um conjunto finito de estados do sistema, alocados nas vizinhanças do ponto de equilíbrio do PISDR.
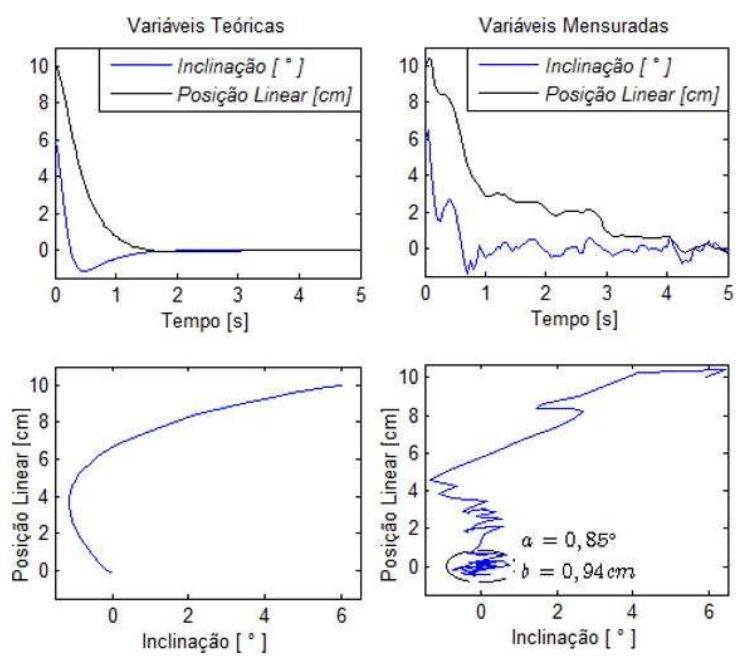

Figura 6. Resposta à condição inicial na realização em espaços de estados para os ganhos de Lyapunov.
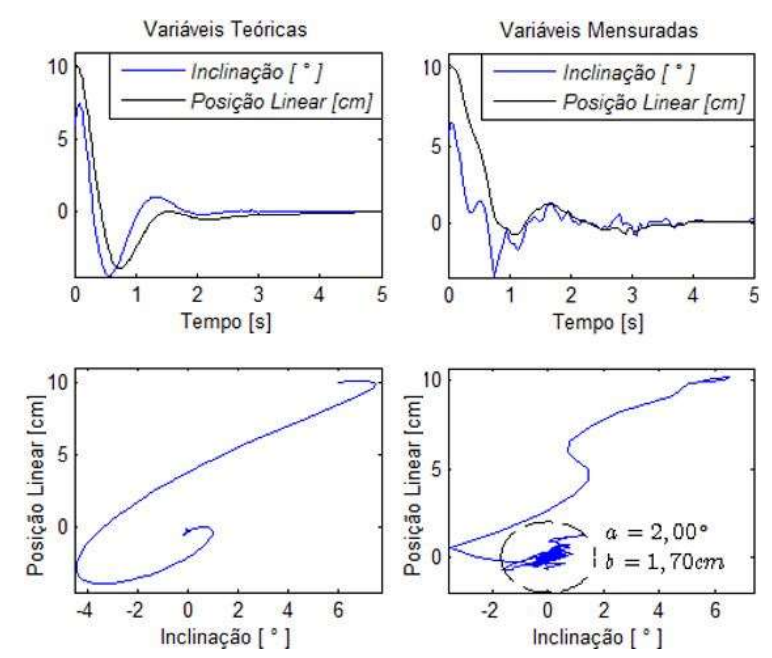

Figura 7. Resposta à condição inicial na implementação da arquitetura de controladores PIDs proposta.

Para o primeiro controlador, implementado com o ganho de realimentação de Lyapunov, o semieixo da elipse paralelo ao eixo de inclinação apresenta magnitude de $0,85^{\circ}$ enquanto que, o segundo semieixo apresenta módulo de $0,94 \mathrm{~cm}$. Analogamente, para a segunda arquitetura de controle proposta, essas magnitudes correspondem a $2,00^{\circ}$ e $1,70 \mathrm{~cm}$, respectivamente.

\subsection{Resposta ao degrau de referência de posição}

As Figuras 8 e 9 ilustram, respectivamente, a resposta do modelo teórico e do sistema real, uma vez especificado o setpoint de posição linear média do PISDR em $13 \mathrm{~cm}$. Ambas as arquiteturas de controle propostas foram capazes de atingir a especificação de referência dada. $O$ erro de posição linear, calculado no intervalo de 8 a 10 s foi de 0,48 e $0,14 \mathrm{~cm}$ respectivamente, em relação as magnitudes mensuradas correspondentes. Percebe-se que, no entanto, a deflexão angular de inclinação do PISDR foi superior a $3^{\circ}$ contrapondo com um dos requisitos estabelecido no projeto dos controladores. 
Quanto à avaliação da ação de controle, observa-se a divergência de amplitude do sinal teórico em relação ao mensurado. Parte desse efeito, deve-se a não contabilização da região de zona morta dos motores elétricos no sinal mensurado. Testes realizados com o PISDR na posição vertical, constataram a rotação dos motores elétricos para um sinal de tensão elétrica com amplitude superior $3,13 \mathrm{~V}$.
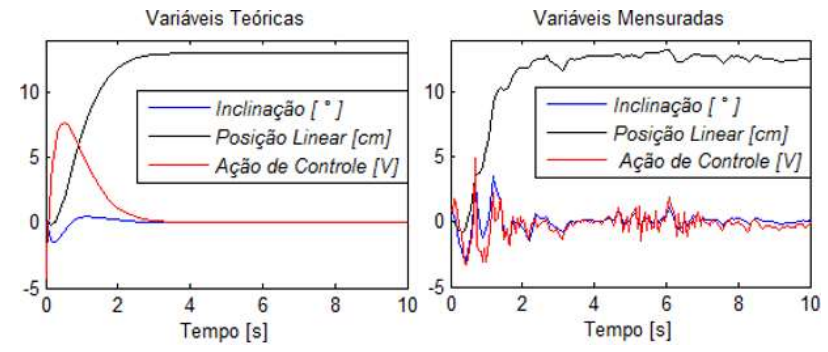

Figura 8. Resposta ao degrau de posição na implementação de controle em espaço de estados, a partir dos ganhos de Lyapunov.
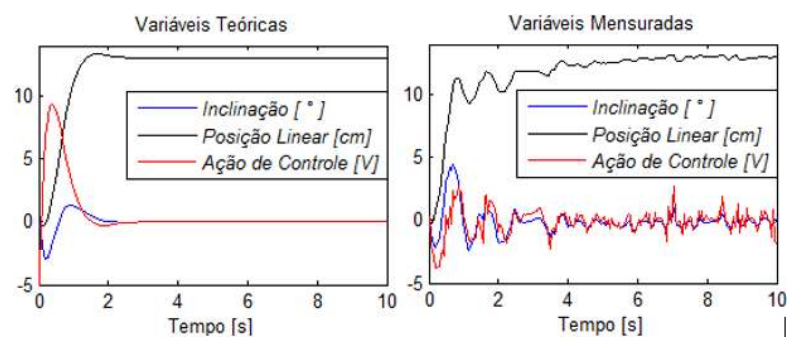

Figura 9. Resposta ao degrau de posição na implementação da arquitetura de controladores PIDs proposta.

Observa-se que, para ambos os cenários de teste, há uma diferença entre modelo teóricos e o real, provenientes de nãolinearidades, como folga nas engrenagens da redução do sistema motor-roda, assim como ruído de medição, visto que os componentes utilizados no protótipo são de baixo custo.

\section{CONCLUSÕES}

As características dinâmicas do sistema em estudo foram descritas pelo modelo matemático obtido, mostrando-se uma alternativa válida e consistente para análise da dinâmica física do sistema, servindo de base teórica para elucidação de técnicas de controle que atendam os objetivos propostos. Neste caso, a principal contribuição do trabalho é relacionada a apresentação de ferramentas para modelagem matemática e validação de um sistema do tipo PISDR, e sua implementação.

De modo geral os controladores projetados foram eficazes na resolução do problema de equilíbrio do PISDR. Destaca-se a relevância desse estudo em problemas similares que envolvam situações de modelagem e projeto de controladores lineares que garantam a estabilidade assintótica para o estado de mínima energia do sistema.

As arquiteturas de controle implementadas possibilitaram a constatação da existência de uma região no domínio dos estados do sistema, contendo a origem, donde é possível caracterizar a convergência assintótica dos estados para o ponto de equilíbrio do sistema. Essa região é conhecida na literatura pela denominação de conjunto atrator (Milhomem, 2013). Uma avaliação preliminar da resposta dos estados do sistema apresentados nas figuras a Seção 4, permite inferir a existência de um sistema de fase não-mínima alterando significativamente a resposta transitória do PISDR. Em sistemas de fase não-mínima a resposta inicia-se em direção contrária à final, relacionada a presença de um zero no SPD, que contribui com atraso de fase (Aguirre, 2007).

Ainda, o problema de equilíbrio do PISDR pode ser aperfeiçoado para tratar situações de modelagem e controle que envolvam variações de parâmetros de inércia do sistema. Como é caso, de veículos de equilíbrio tripulados. Nesse sentido, há possibilidade de averiguar a eficácia na aplicação de outros controladores, visando um aumento de robustez e garantia das condições de estabilidade do sistema.

\section{AGRADECIMENTOS}

Ao Instituto Federal de Santa Catarina que possibilitou este trabalho a ser desenvolvido e publicado neste evento.

\section{REFERÊNCIAS}

Aguirre, L. A. (2007). Introdução à Identificação de Sistemas - Técnicas Lineares e Não-Lineares Aplicadas a Sistemas Reais, 3 edn, Editora UFMG.

Boyd, S., Ghaoui, L. E., Feron, E. and Balkrishnan, V. (1994). Linear Matrix Inequalities in System and Control Theory, PA: SIAM Studies in Applied Mathematics, Philadelphia.

Chen, C. T. (1999). Linear System and Design. 3 edn, Oxford University Press, Inc.

Frizon, L. J.; Peccin, V. B.; Jinbo, M. (2015). Estimação dinâmica de ângulo baseado em giroscópio e acelerômetro com filtro de Kalman. Instituto Federal de Santa Catarina, Santa Catarina, 2015.

Jung, S. and Kim, S. S. (2008). Control Experiment of a Wheel-Driven Mobile Inverted Pendulum UsingNeural Network. IEEE Transactions on Control Systems Technology, 16: 297-303.

Khalil, H. K. (2002). Nonlinear Systems. 3. ed. New Jersey: Prentice Hall.

Lathi, B. (2007). Sinais e sistemas lineares, 2 edn, Bookman.

Milhomem, R. L. (2013). Análise e síntese de sistemas sujeitos a uma classe de perturbações limitadas e aplicações. Universidade Federal de Santa Catarina, 2013.

Nawawi, S. W., Ahmad, M. N., Osman, J. H. S., (2008). Realtime control of a two-wheeled inverted pendulum mobile robot. International Journal of Computer and Information Engineering, 70-76.

Ogata, K (2011). Engenharia de Controle Moderno, 5 edn, Pearson Prentice Hall.

Vendramini, G.; Silva, P. S. (2010). Controle de um pêndulo invertido sobre uma plataforma móvel utilizando PID e MFCA. $9^{\text {th }}$ Brazilian Conference on Dynamics, Control and their Applications.

Segway inc., Simply Moving. Disponível em: $<$ http://www.segway.com>. Acesso em 5 mar. 2018. 